\title{
Identification of TMPRSS2-ERG mechanisms in prostate cancer invasiveness: Involvement of MMP-9 and plexin B1
}

\author{
BIDE LIU, XIAO GU, TIANBAO HUANG, YANG LUAN and XUEFEI DING \\ Department of Urology, Clinical Medical College, Yangzhou University, Yangzhou, Jiangsu 225001, P.R. China
}

Received May 3, 2016; Accepted September 6, 2016

DOI: $10.3892 /$ or.2016.5277

\begin{abstract}
The relationship of TMPRSS2-ERG fusion gene with matrix metalloproteinase-9 (MMP-9) and PLXNB1 (plexin B1) in regulation of prostate cancer (PCa) aggressiveness was investigated. Fluorescence in situ hybridization (FISH) assays, qRT-PCR and western blot analysis were employed to detect the expression of TMPRSS2-ERG fusion gene, ERG, MMP-9 and PLXNB1 of 135 human tissues, which included 55 metastatic PCa cases, 50 localized PCa cases and 30 BPH cases. Then using siRNA (anti-ERG, MMP-9 and PLXNB1, respectively) downregulation of the target gene of VCaP and PC-3 cells, MTT and Transwell were performed. The results showed that the positive rate of TMPRSS2-ERG fusion was $38.1 \%(40 / 105)$ in total PCa samples, 47.3\% (26/55) of metastatic PCa, $28.0 \%$ (14/50) of localized PCa, while $0.0 \%$ $(0 / 30)$ in BPH samples. The mRNA and protein expression of ERG, MMP-9 and PLXNB1 were higher in metastatic PCa $(\mathrm{P}<0.0001)$, and the mRNA expression of the three genes were positively correlated with TMPRSS2-ERG fusionin PCa group $(\mathrm{P}<0.0001)$. siRNA transfected $\mathrm{PCa}$ cells can effectively downregulate the target gene expression, and we identified that MMP-9 and PLXNB1 expression were all regulated by TMPRSS2-ERG fusion gene. While only PLXNB1 contributed to TMPRSS2-ERG mediated enhancements of VCaP cell migration and invasion. The results demonstrated that PLXNB1, but not MMP-9, was the target gene directly related to TMPRSS2-ERG in PCa cell migration and invasion.
\end{abstract}

\section{Introduction}

Prostate cancer (PCa) is the second most prevalent cancer and the fifth leading cause of cancer-related death affecting men worldwide (1). Recent genomic and oncogenetic study involved in $\mathrm{PCa}$ onset and progression highlighted several

Correspondence to: Dr Xuefei Ding, Department of Urology, Clinical Medical College, Yangzhou University, 98 West Nantong Road, Yangzhou, Jiangsu 225001, P.R. China

E-mail: p15161848813@163.com

Key words: prostate cancer, TMPRSS2-ERG, matrix metalloproteinase-9, PLXNB1, invasiveness genomic alterations. Among these, gene fusion of the androgen-regulated trans-membrane-serine protease gene (TMPRSS2) and erythroblast transformation-specific (ETS) family members are considered as hallmarks, and the fusion of ETS-related gene (ERG) with TMPRSS2 is the most frequent (2). TMPRSS2-ERG fusion gene leads to overexpression of ERG, and plays an important role in PCa progression and invasiveness (2).

The function of TMPRSS2-ERG in PCa has been explored using PCa cell lines, human tissues and mouse models. Some research demonstrated that aberrant TMPRSS2-ERG expression in mouse prostate can induce prostatic intraepithelial neoplasia (PIN), and a study showed that patients with ERG-positive high-grade PIN are much more likely to progress to $\mathrm{PCa}(3,4)$. While others have highlighted that TMPRSS2-ERG expression cooperates with or induces other genomic alterations, such as PDE4D7 or EZH2 overexpression, PTEN deletion, PI3K pathway, or androgen receptor (AR) signaling to promote $\mathrm{PCa}$ genesis, progression, migration and invasion (5-8). These studies provide functional insight into the role of TMPRSS2-ERG in PCa.

Matrix metalloproteinases (MMPs), especially MMP-9, have well-recognized roles in cancer metastasis and invasion. Plexins (PLXNs) are a family of transmembrane receptors for semaphorins, there were investigations showing that overexpression of PLXNB1 can activate ErbB2, hinder Rac and R-Ras binding, inhibit R-Ras GAP activity, or promote AR translocation to the nucleus to increase the motility and invasion of PCa $(9,10)$. Given the potential ability of TMPRSS2-ERG fusion gene in invasiveness, we hypothesized that MMP-9 and/or PLXNB1 might be the target gene of TMPRSS2-ERG to promote $\mathrm{PCa}$ invasion and metastasis.

In this study, we therefore aimed to characterize the relation of TMPRSS2-ERG and MMP-9 as well as PLXNB1 in regulation of $\mathrm{PCa}$ aggressiveness. Additionally, the expression and clinicopathological association of the three genes of Chinese PCa patients were also investigated.

\section{Materials and methods}

Clinical samples. Our study consisted of 55 needle biopsy tissues of bone metastatic $\mathrm{PCa}, 50$ radical resection tissues from localized $\mathrm{PCa}$, and 30 electrosection tissues of benign prostatic hyperplasia (BPH). None of the patient received preoperative radiation or androgen deprivation therapy. All 
the cases were collected from 2009-2015 at Northern Jiangsu People's Hospital (Jiangsu, China), and approved by the local ethics committee of Northern Jiangsu People's Hospital, in accordance with the guidelines of the 1975 Declaration of Hesinki. Patients' clinicopathological variables are shown in Table I. Tissues were collected directly from the operating room, part of the tissue from each case was immersed in formalin immediately, and the rest stored in liquid nitrogen.

Fluorescence in situ hybridization (FISH) analysis. Four-micrometer $(4-\mu \mathrm{m})$ sections of the formalin-fixed, paraffin-embedded tissues were cut for FISH analysis. TMPRSS2-ERG fusion was tested by a dual-color dual-fusion model probes (Beijing GP Medical Technologies, Ltd., Beijing, China) according to the manufacturer's protocol. Slides were examined using ImagingZ1 microscope (Carl Zeiss, Oberkochen, Germany). FISH signals were scored manually (100X oil immersion) in morphologically intact and non-overlapping nuclei. The positive signal patterns are one yellow/one green in a cell, which revealed a deletion pattern of fusion, or one yellow/one green/one red in a cell, which illustrated an insertion pattern. Additionally, the negative signal pattern is two yellow signals in a cell. The criteria to determine a PCa case TMPRSS2-ERG fusion-positive was that $\geq 20$ cells with the positive signal pattern in a random count of 100 cells.

Cell culture. Human PCa cell lines VCaP (TMPRSS2-ERG fusion-positive) and PC-3 (TMPRSS2-ERG fusion-negative) were obtained from Chinese Academy of Sciences Typical Culture Preservation Committee Cell Bank and maintained in RPMI-1640 and Ham's/F12 medium (Hyclone Laboratories Inc., Logan, UT, USA) respectively, and supplemented with $10 \%$ fetal bovine serum (Clark Bioscience, USA) at $37^{\circ} \mathrm{C}$ in a humidified $5 \% \mathrm{CO}_{2}$ incubator.

siRNA-mediated knockdown. VcaP and PC-3 cells were seeded at a density of $5 \times 10^{5}$ cells/well into 6 -well plates and cultured overnight at $37^{\circ} \mathrm{C}$ with $5 \% \mathrm{CO}_{2}$ until the cells reached $70-80 \%$ confluency. Small interfering RNA (siRNA) transfection on two cell lines was carried out using Lipofectamine 2000 (Invitrogen, Carlsbad, CA, USA) according to the manufacturer's protocol. Specific siRNA sequences targeting the human ERG, MMP-9, and PLXNB1 were all designed and synthesized respectively (GenePharma Pharmaceutical Co., Shanghai, China), and the sequences are provided in Table II. The NC group was defined as negative control, and the mock group was the ones supplemented with the transfection reagent only.

Cell proliferation, migration and invasion assays. Cell proliferation was determined using MTT assay in 96-well plate $\left(1 \times 10^{4}\right.$ cells/well). After transfection for $12,24,48$ or $72 \mathrm{~h}$, at $10 \mu \mathrm{l} /$ well MTT solution $(5 \mathrm{mg} / \mathrm{ml})$ was added and incubated for $4 \mathrm{~h}$. The supernatant was removed and DMSO (100 $\mu \mathrm{l} /$ well) added. The absorbance [estimated by optical density (OD)] was measured at $492 \mathrm{~nm}$ using Epoch Microplate Spectrophotometer (BioTek, VT, USA).

Cell migration assays were performed with $1 \times 10^{5}$ cells in a serum-free medium and seeded on the upper chamber of Transwell inserts with a pore size of $8 \mu \mathrm{m}$. While the cell
Table I. Clinicopathological variables of patient sample (mean \pm standard deviation).

\begin{tabular}{lccc}
\hline & $\begin{array}{c}\text { Metastatic PCa } \\
(\mathrm{n}=55)\end{array}$ & $\begin{array}{c}\text { Localized PCa } \\
(\mathrm{n}=50)\end{array}$ & $\begin{array}{c}\mathrm{BPH} \\
(\mathrm{n}=30)\end{array}$ \\
\hline Age (years) & $70.8 \pm 5.8$ & $70.2 \pm 5.8$ & $68.1 \pm 7.7$ \\
PSA (ng/ml) & $36.3 \pm 34.4$ & $27.5 \pm 27.1$ & $6.7 \pm 6.6$ \\
Volume $\left(\mathrm{cm}^{3}\right)$ & $56.3 \pm 29.5$ & $44.1 \pm 21.6$ & $66.4 \pm 57.8$ \\
Gleason score & & & \\
$\leq 6$ & $3(5.5 \%)$ & $10(26.0 \%)$ & - \\
7 & $16(29.1 \%)$ & $20(44.0 \%)$ & - \\
$\geq 8$ & $36(65.4 \%)$ & $20(30.0 \%)$ & - \\
Pathological & & & \\
tumor stage & & & - \\
2 & - & $33(66 \%)$ & - \\
3 & - & $12(24 \%)$ & - \\
4 & - & $5(10 \%)$ & - \\
\hline
\end{tabular}

invasion assays were performed on Matrigel-coated upper chamber with $1 \times 10^{5}$ cells, the culture medium containing $10 \%$ FBS as a chemoattractant was added to the lower chamber. After 24-h incubation, cells were fixed and labeled with crystal violet, and the migrated or invaded cells were counted in five randomly selected microscopic fields under x 200 microscopy (Olympus, Tokyo, Japan).

RNA extraction and quantitative real-time PCR ( $q R T-P C R)$. Total RNA isolated from human prostate tissues and cells was extracted using TRIzol reagent according to the manufacturer's instructions (Invitrogen). cDNA was reverse transcribed from $1 \mu \mathrm{g}$ total RNA using PrimeScript ${ }^{\mathrm{TM}} \mathrm{RT}$ Master Mix kit (Takara, Dalian, China) according to the manufacturer's protocol. qRT-PCR was performed with the SYBR Premix Ex Taq (Takara). All primer sequences are listed in Table II. Relative mRNA expression levels were calculated by the comparative $2^{-\Delta \Delta \mathrm{Ct}}$ method.

Western blot analysis. We randomly selected 10 cases from each group of patients to detect the protein expression. Total protein of human prostate tissues and PCa cell lines was extracted using RIPA lysate buffer (Beyotime, Shanghai, China) and the concentration was quantified by BCA protein assay kit (Beyotime). Protein lysates (20 $\mu$ g per lane) were separated by sodium dodecyl sulfate (SDS)-polyacrylamide gel electrophoresis and transferred onto nitrocellulose membranes. After blocking with 5\% fat-free milk, the membranes were incubated with primary antibodies at $4^{\circ} \mathrm{C}$ overnight, followed by horseradish peroxidase-conjugated secondary antibodies. Immunoreactive bands were visualized using FluorChem FC2 (Alpha Innotech, CA, USA). The primary antibodies to ERG, MMP-9 and PLXNB1 were all purchased from Abcam (Cambridge, MA, USA), and were diluted 1,000, 5,000 and 5,000 times respectively. The internal reference GaPDH was from KangChen Biotech (Shanghai, China) and diluted 5,000 times. 
Table II. The sequences of primers and siRNA.

\begin{tabular}{lll}
\hline Gene & \multicolumn{1}{c}{ Forward primer } & Reverse primer \\
\hline ERG & 5'-GTG CCA AAC ATC CTA TTT CC-3' & 5'-CAT TTA TAC ACT ACG AGT TG-3' \\
MMP-9 & 5'-CCT TCT ACG GCC ACT ACT GT-3' & 5'-CAC TTG TCG GCG ATA AGG AA-3' \\
PLXNB1 & 5'-TCT GCT CAG TGA CCT GGT TG-3' & 5'-CTA CGG AGT CCC TCA CGA AG-3' \\
$\beta$-actin & 5'-GGG ACC TGA CTG ACT ACC TC -3' & 5'-TCA TAC TCC TGC TTG CTG AT -3' \\
ERG siRNA & 5'-CGA CAU CCU UCU CUC ACA UAU-3' & 5'-UGA UGU UGA UAA AGC CUU A-3' \\
MMP-9 siRNA & 5'-CUA UGG UCC UCG CCC UGA ATT-3' & 5'-UUC AGG GCG AGG ACC AUA GAG-3' \\
PLXNB1 siRNA & 5'-AAG GUA UAC AGA CAG AUG GAC AUC C-3' & 5'-AAG CUC GAA AUA UCU CCU-3' \\
Negative siRNA & 5'-UUC UCC GAA CGU GUC ACG UTT-3' & 5'-ACG UGA CAA GUU CGG AGA ATT-3'
\end{tabular}
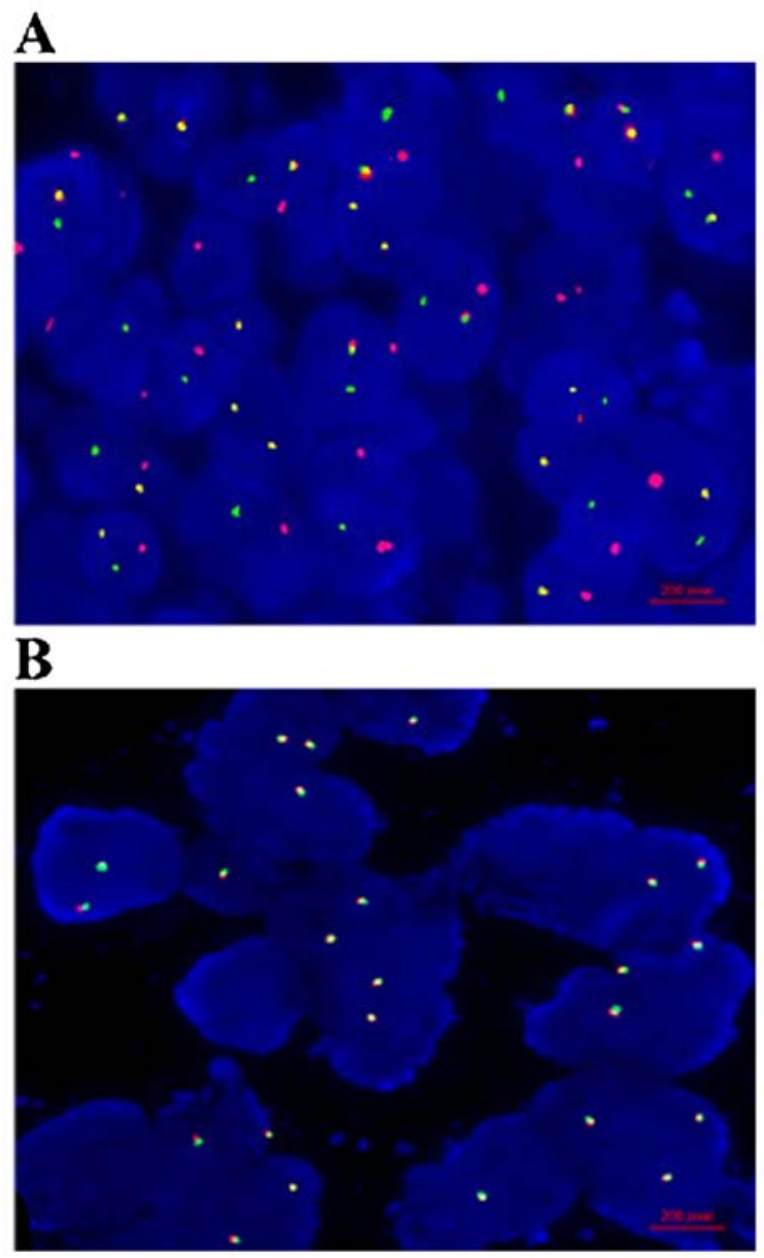

Figure 1. The expression of TMPRSS2-ERG fusion gene in human tissues (A) Representative image for TMPRSS2-ERG fusion-positive, one yellow/ one green/one red signals. (B) Representative image for TMPRSS2-ERG fusion-negative, two yellow signals.

Statistical analysis. Statistical analysis was performed using SPSS 17.0 software (IBM Corp., NY, USA), with a significance level of 0.05 (two-tailed probability). Data were expressed as percentage or means \pm standard deviation. The distributions of continuous and categorical variables between cases and controls were compared using Student's t-test and one-way ANOVA analysis. Correlations between the clinicopathological parameters and gene expression were determined by Chi-square test and rank correlation analysis.

\section{Results}

MMP-9 and PLXNBI expression were associated with TMPRSS2-ERG expression in human PCa tissues. To investigate whether MMP-9 and PLXNB1 are target genes of TMPRSS2-ERG in PCa, we first examined their expression in human samples. A total of 135 cases were successfully analyzed by FISH, qRT-PCR, and 30 randomly selected cases by western blot analysis. Overall, TMPRSS2-ERG fusion was positive in $47.3 \%(26 / 55)$ of metastatic PCa, $28.0 \%$ (14/50) of localized $\mathrm{PCa}$, and $0.0 \%(0 / 30)$ of BPH (Fig. 1). The mRNA expression of ERG, MMP-9 and PLXNB1 were increasing gradually from $\mathrm{BPH}$ to metastatic PCa, as well as protein expression (Figs. 2 and 3). Additionally, in metastatic PCa cohort, localized PCa cohort and total PCa cohort, the mRNA expression levels of ERG, MMP-9 and PLXNB1 were all significantly higher in TMPRSS2-ERG-positive PCa samples than in TMPRSS2ERG-negative samples (P-values were all <0.0001).

The mRNA levels of ERG, MMP-9 and PLXNB1 were all positively correlated with TMPRSS2-ERG fusion $(\mathrm{P}<0.0001)$, in metastatic PCa group the spearman values were 0.757 , 0.833 and 0.865 respectively, in the localized $\mathrm{PCa}$ group were $0.778,0.781$ and 0.889 , respectively, and in total PCa group were $0.549,0.806$ and 0.626 , respectively. Moreover, the mRNA expression of MMP-9 and PLXNB1 were positively correlated with ERG mRNA level $(\mathrm{P}<0.0001)$, in metastatic PCa group the Spearman values were 0.705 and 0.751 , in localized PCa group were 0.640 and 0.710 , and in total PCa group were 0.762 and 0.919 . While there was no relationship between MMP-9 and PLXNB1 in each of the PCa groups. Furthermore, there was no relationship among the genes with each other in BPH samples.

Chi-square test demonstrated that high Gleason score patients tend to the fusion of TMPRSS2 and ERG gene in metastatic $\mathrm{PCa}$ group $(\mathrm{P}=0.007)$ and total $\mathrm{PCa}$ group $(\mathrm{P}=0.039)$. The relationship between gene expression and clinicopathological parameters is shown in Table III. Obviously, there was no relationship in metastatic PCa samples. While in localized PCa group, TMPRSS2-ERG fusion gene positive rate was positively correlated with serum PSA level, Gleason score and pathological tumor stage, additionally, the Gleason score and 


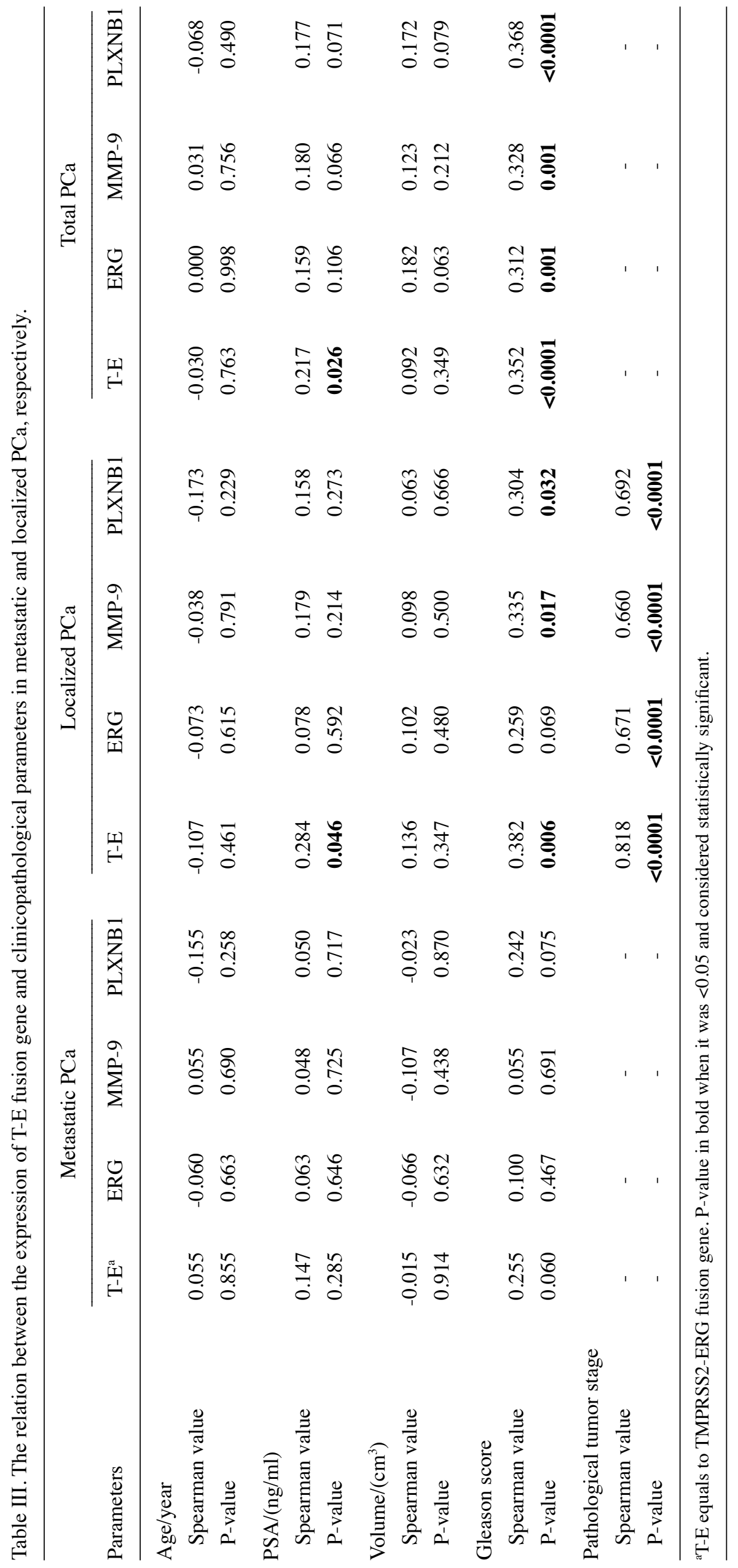



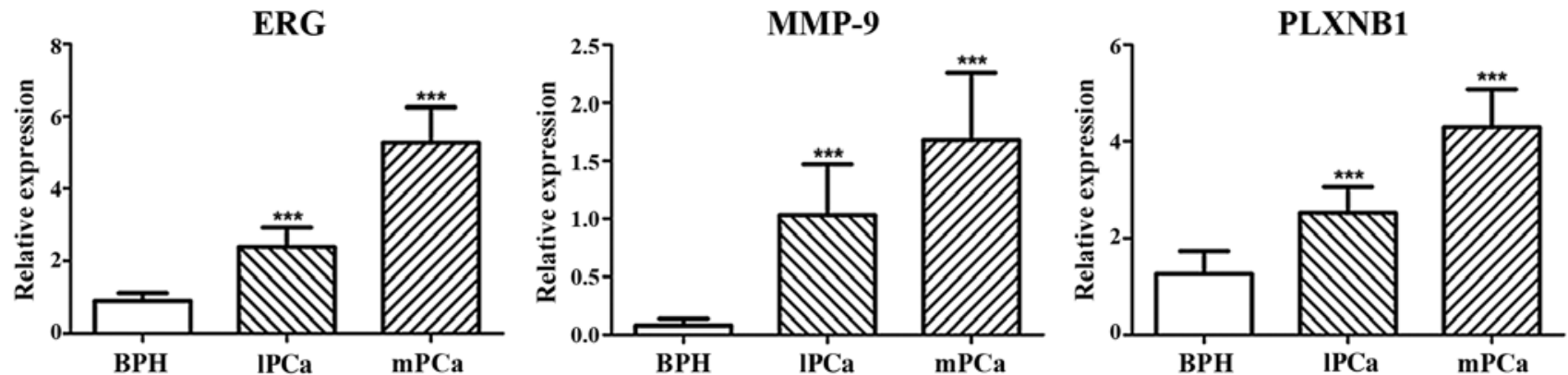

Figure 2. The mRNA expression of ERG, MMP-9 and PLXNB1 in human tissues. ${ }^{* * *} \mathrm{P}<0.001$. mPCa and 1PCa represent metastatic PCa and localized PCa, respectively.

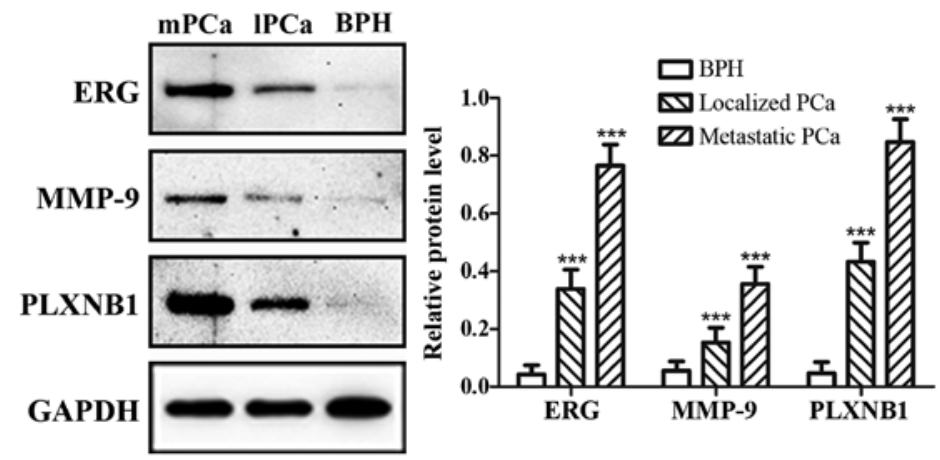

Figure 3. The protein expression of ERG, MMP-9 and PLXNB1 in human tissues. ${ }^{* * *} \mathrm{P}<0.001, \mathrm{n}=10$. $\mathrm{mPCa}$ and $1 \mathrm{PCa}$ represent metastatic PCa and localized PCa, respectively.
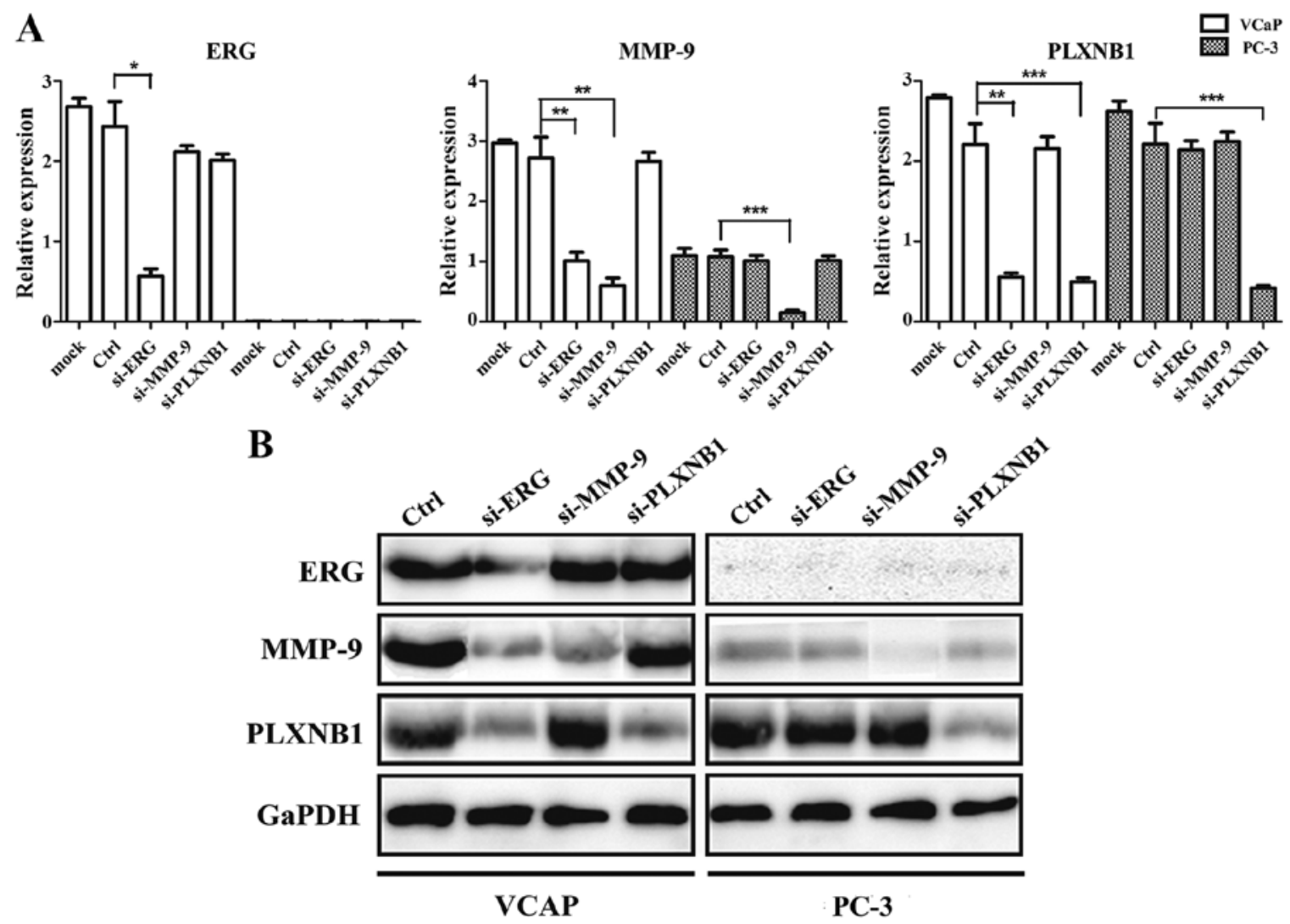

Figure 4. The mRNA and protein expression of target genes in PCa cell lines after siRNA transfection. (A) Expression of ERG was downregulated by si-ERG in VCaP cells; MMP-9 expression was decreased by si-ERG and si-MMP-9 in VCaP cells, while just decreased by si-MMP-9 in PC-3 cells; PLXNB1 was downregulated by si-ERG and si-PLXNB1 in VCaP cells, but just decreased by si-PLXNB1 in PC-3 cells. (B) Protein expression of target genes in VCaP and PC-3 cells. ${ }^{*} \mathrm{P}<0.05,{ }^{* * *} \mathrm{P}<0.01$ and ${ }^{* * *} \mathrm{P}<0.001$. 

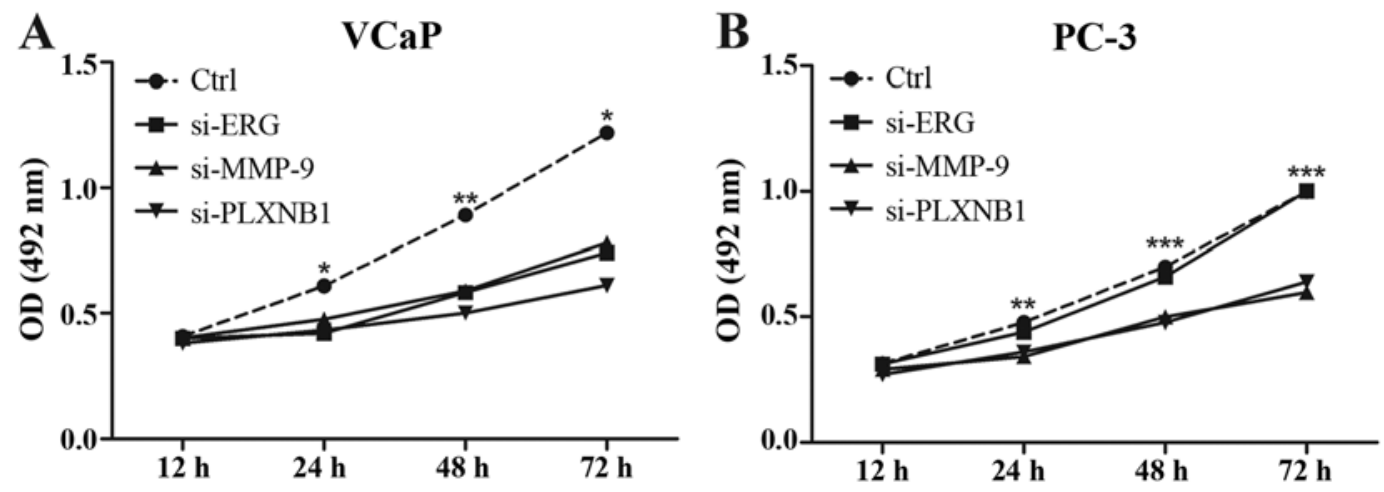

Figure 5. The change of proliferation capacity in PCa cells after siRNA transfection. (A) Knockdown of ERG, MMP-9 and PLXNB1 can significantly induce apoptosis in VCaP cells. (B) Knockdown of MMP-9 and PLXNB1 can significantly induce apoptosis in PC-3 cells. ${ }^{*} \mathrm{P}<0.05$, ${ }^{* * *} \mathrm{P}<0.01$ and ${ }^{* * * *} \mathrm{P}<0.001$.
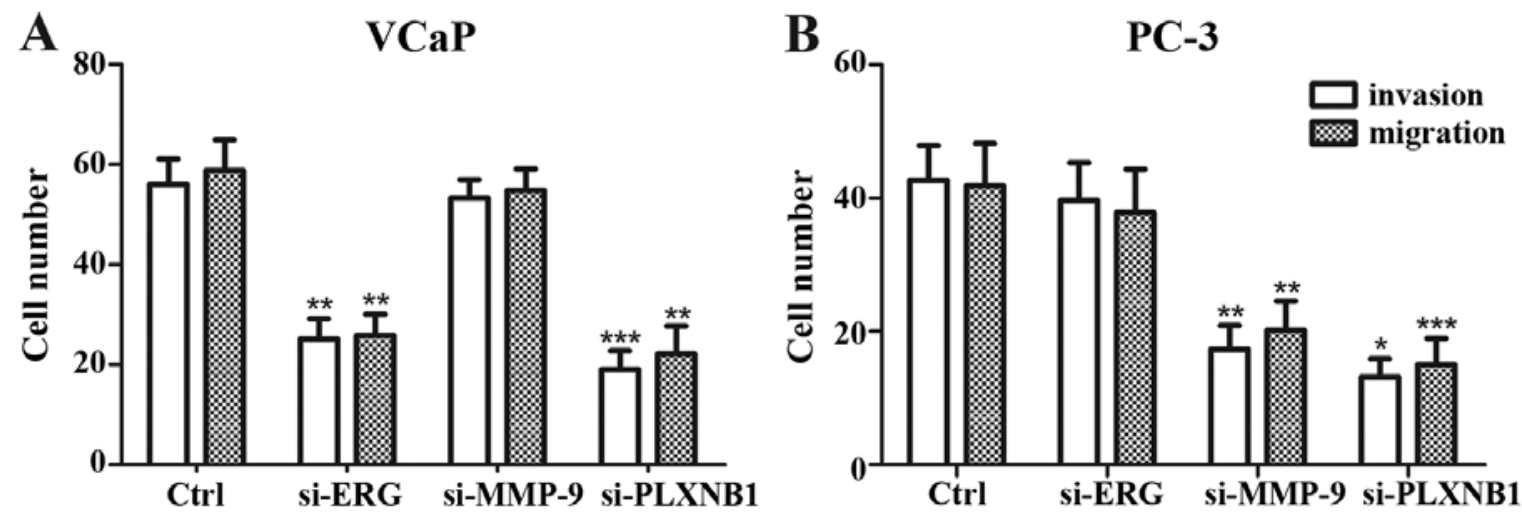

Figure 6. The change of migration and invasion capacity in PCa cells after siRNA transfection. (A) Knockdown of ERG and PLXNB1 can significantly decrease the migration and invasive capacity in VCaP cells. (B) Knockdown of MMP-9 and PLXNB1 can significantly decrease the migration and invasive capacity in PC-3 cells. ${ }^{*} \mathrm{P}<0.05,{ }^{* *} \mathrm{P}<0.01$ and ${ }^{* * *} \mathrm{P}<0.001$.

pathological tumor stage were positively correlated with the expression of MMP-9 and PLXNB1. In total PCa samples, the Gleason score was positively correlated with the expression of each gene, and serum PSA level was positively correlated with TMPRSS2-ERG fusion gene positive rate.

Successful transformation of siRNA to PCa cells and inhibition of the expression of target genes. To determine the role of TMPRSS2-ERG fusion gene, MMP-9 and PLXNB1 in the invasiveness of PCa cells, specific siRNA targeting of ERG, MMP-9 and PLXNB1 were transiently transfected into PCa cells, and, the mRNA and protein of the target genes were detected. qRT-PCR and western blot analysis confirmed the downregulation of ERG, MMP-9 and PLXNB1 in VCaP cells, and downregulation of MMP-9 and PLXNB1 in PC-3 cells, compared to the cells transfected with negative siRNA (Fig. 4). We also discovered that downregulated ERG expression can significantly decrease the mRNA and protein expression of MMP-9 and PLXNB1 in VCaP cells.

The effects of siRNA on proliferation, migration and invasion in PCa cells. Consistent with previous findings, the mRNA and protein expression level of ERG were high in PCa cell line VCaP, but almost no expression was observed in PC-3 cells (Fig. 4). Using MTT assay, we found that ERG, MMP-9 and
PLXNB1 knockdown VCaP cells have higher apoptosis rate (Fig. 5A); while only MMP-9 and PLXNB1 knockdown have higher apoptosis rate in PC-3 cells (Fig. 5B).

In VCaP cells, siRNA knockdown of ERG significantly decreased migration and invasive capacity $(\mathrm{P}=0.002$ and 0.008 respectively, Fig. 6A), similarly, knockdown of PLXNB1 also reduced migration and invasive capacity $(\mathrm{P}=0.005$ and $<0.0001$ respectively, Fig. 6A). In PC-3 cells, knockdown of MMP-9 downregulated the migration and invasive capacity significantly $(\mathrm{P}=0.002$ and 0.007 respectively, Fig. 6B); and knockdown of PLXNB1 observably decreased the migration and invasive ability ( $\mathrm{P} \leq 0.0001$ and 0.015 respectively, Fig. 6B).

\section{Discussion}

The mechanism and treatment of metastatic PCa have been the focus of targeted therapy as a new treatment with great application prospects. Santoni et al indicated that fully understanding the role of TMPRSS2-ERG is important for individualised therapy in PCa patients (11). Urbinati et al designed a kind of siRNA anti-TMPRSS2-ERG and successfully downregulated the expression of this fusion gene, and observably inhibited the proliferation of PCa cells (12). The above showed the potential and importance of TMPRSS2-ERG in PCa targeting treatment. 
Here, using human samples, we showed that, as expected, the positive rate of TMPRSS2-ERG fusion in human prostate tissues was increasing with the degree of invasiveness. Additionally, this fusion can obviously upregulate the mRNA and protein expression of ERG, as well as MMP-9 and PLXNB1. These findings are in agreement with the role of TMPRSS2-ERG as revealed using various PCa cell models $(2,10,13)$. The positive rate of TMPRSS2-ERG fusion detected by FISH in our study was $38.1 \%$ (40/105). While, Magi-Galluzzi et al reported that TMPRSS2-ERG fusion was present in $50.0 \%$ (21/42) of Caucasian, 31.3\% (20/64) of African-American, and 15.9\% (7/44) of Japanese, and Dong et al demonstrated the incidence was $14.3 \%$ (13/91) by biopsy specimens or $11.1 \%(2 / 18)$ by radical prostatectomy samples $(14,15)$. The reasons why the frequency of gene fusion varies among different studies is complex, probably owing to race, methods of obtaining specimens, the sensitivity of the technique used, the number of samples included in the study, the criteria used to determined a positive signal and the patients from different areas.

MMP-9 has been confirmed to be correlated with increased invasion and metastasis in various tumor types, including PCa (16). In fact, several transcription factors, such as twist-related protein 1 (Twist1), high-mobility group protein A2 (HMGA2), mitogen-activated protein kinase 1 (MAPK1), and signal transducer and activator of transcription 3 (STAT3), have been shown to control MMP-9 expression in numerous cancer types (17-20). Here, we showed that MMP-9 expression was correlated with TMPRSS2-ERG as well as ERG in PCa samples, and was positively regulated by ERG which is upregulated by TMPRSS2-ERG fusion in VCaP cells. Additionally, it has been reported that ETS-1 promotes the invasiveness of paclitaxel-resistant and hormone-refractory PCa cells by increasing MMP-9 expression, moreover, a recent study demonstrated that overexpression of ETV4 can upregulate the invasiveness of PCa through increasing MMP-9 expression $(21,22)$. ERG is not only a transcription factor, but also a member of the ETS family, so these findings emphasized the importance of ETS in controlling MMP-9 expression in PCa. However, knockdown of the expression of MMP-9 did not weaken TMPRSS2-ERGinduced cell migration or invasion in $\mathrm{VCaP}$ cells. This result was similar to that treating TMPRSS2-ERG expressing PCa cells with MMP-9 inhibitor (23). Therefore, future studies are required to investigate the role of MMP-9 in TMPRSS2-ERGpositive PCa cells using in vivo models.

PLXNs activate many characteristics of the invasive phenotype seen in cancer progression, inducing changes in extracellular matrix (ECM) adhesion, motility, scatter, and branching morphogenesis (24). It was revealed that PLXNA2 expression was higher in more aggressive breast cancer cell types, and another study showed that TMPRSS2-ERG positively and directly regulates PLXNA2 expression in PC $3 \mathrm{c}$ cells $(13,25)$. Ye et al found that PLXNB1 was significantly higher expressed in serous ovarian carcinomas (26). Our results showed that, similar to MMP-9, PLXNB1 was not only expressed higher in metastatic PCa tissues, but positively and directly regulated by TMPRSS2-ERG fusion in VCaP cells. Together with the finding that downregulation of PLXNB1 in $\mathrm{VCaP}$ cells can decrease the migration and invasion capacity, we first discovered that PLXNB1 contributes, at least in part, to TMPRSS2-ERG-induced VCaP cell migration and invasion. This finding supports the potential of PLXNB1 in PCa metastasis.

The association between TMPRSS2-ERG fusion and PCa clinical outcome has not yet been clearly established. Some studies have shown that this gene fusion is not significantly associated with PCa clinicopathological parameters $(27,28)$. However, other studies have demonstrated that it is associated with higher clinical tumor stages and Gleason scores $(29,30)$. Our results showed that TMPRSS2-ERG is positively associated with Gleason scores and serum PSA level in localized PCa samples and total PCa samples, while the correlation was weak. Additionally, the expression of MMP-9 and PLXNB1 were positively associated with Gleason scores with a weak correlation. Compared to others research, these uncertain results still need support of larger sample numbers.

In conclusion, our data confirmed the important role of TMPRSS2-ERG in PCa invasiveness, and demonstrated that PLXNB1, but not MMP-9, was the target gene directly related to TMPRSS2-ERG in PCa cell migration and invasion, providing novel insights into the role of TMPRSS2-ERG in PCa invasiveness.

\section{Acknowledgements}

This study was supported by the generous funding of the Yangzhou Science and Technology Natural Science Foundation (YZ2014052), and Project of Jiangsu Province Health and Family Planning Commission (H2015550).

\section{References}

1. International Agency for Research on Cancer: Prostate cancer: estimated incidence, mortality, and prevalence worldwide. http://globocan.iarc.fr/Pages/fact_sheets_cancer.aspx. Accessed March 20, 2014.

2. Tomlins SA, Rhodes DR, Perner S, Dhanasekaran SM, Mehra R, Sun XW, Varambally S, Cao X, Tchinda J, Kuefer R, et al: Recurrent fusion of TMPRSS2 and ETS transcription factor genes in prostate cancer. Science 310: 644-648, 2005.

3. Klezovitch O, Risk M, Coleman I, Lucas JM, Null M, True LD, Nelson PS and Vasioukhin V: A causal role for ERG in neoplastic transformation of prostate epithelium. Proc Natl Acad Sci USA 105: 2105-2110, 2008.

4. Park K, Dalton JT, Narayanan R, Barbieri CE, Hancock ML, Bostwick DG, Steiner MS and Rubin MA: TMPRSS2:ERG gene fusion predicts subsequent detection of prostate cancer in patients with high-grade prostatic intraepithelial neoplasia. J Clin Oncol 32: 206-211, 2014

5. Böttcher R, Henderson DJ, Dulla K, van Strijp D, Waanders LF, Tevz G, Lehman ML, Merkle D, van Leenders GJ, Baillie GS, et al: Human phosphodiesterase 4D7 (PDE4D7) expression is increased in TMPRSS2-ERG-positive primary prostate cancer and independently adds to a reduced risk of post-surgical disease progression. Br J Cancer 113: 1502-1511, 2015.

6. Fallahabadi ZR, Noori Daloii MR, Mahdian R, Behjati F, Shokrgozar MA, Abolhasani M, Asgari M and Shahrokh H: Frequency of PTEN alterations, TMPRSS2-ERG fusion and their association in prostate cancer. Gene 575: 755-760, 2016.

7. King JC, Xu J, Wongvipat J, Hieronymus H, Carver BS, Leung DH, Taylor BS, Sander C, Cardiff RD, Couto SS, et al: Cooperativity of TMPRSS2-ERG with PI3-kinase pathway activation in prostate oncogenesis. Nat Genet 41: 524-526, 2009.

8. Chen Y, Chi P, Rockowitz S, Iaquinta PJ, Shamu T, Shukla S, Gao D, Sirota I, Carver BS, Wongvipat J, et al: ETS factors reprogram the androgen receptor cistrome and prime prostate tumorigenesis in response to PTEN loss. Nat Med 19: 1023-1029, 2013. 
9. Damola A, Legendre A, Ball S, Masters JR and Williamson M: Function of mutant and wild-type plexinbl in prostate cancer cells. Prostate 73: 1326-1335, 2013.

10. Williamson M, de Winter P and Masters JR: Plexin-B1 signalling promotes androgen receptor translocation to the nucleus. Oncogene 35: 1066-1072, 2016.

11. Santoni M, Scarpelli M, Mazzucchelli R, Lopez-Beltran A, Cheng L, Epstein JI, Cascinu S, Briganti A, Catto JW, Montorsi F, et al: Current histopathologic and molecular characterisations of prostate cancer: towards individualised prognosis and therapies. Eur Urol 69: 186-190, 2016.

12. Urbinati G, Ali HM, Rousseau Q, Chapuis H, Desmaële D, Couvreur P and Massaad-Massade L: Antineoplastic effects of siRNA against TMPRSS2-ERG junction oncogene in prostate cancer. PLoS One 10: e0125277, 2015.

13. Tian TV, Tomavo N, Huot L, Flourens A, Bonnelye E, Flajollet S, Hot D, Leroy X, de Launoit Y and Duterque-Coquillaud M: Identification of novel TMPRSS2:ERG mechanisms in prostate cancer metastasis: involvement of MMP9 and PLXNA2. Oncogene 33: 2204-2214, 2014.

14. Magi-Galluzzi C, Tsusuki T, Elson P, Simmerman K, LaFargue C, Esgueva R, Klein E, Rubin MA and Zhou M: TMPRSS2-ERG gene fusion prevalence and class are significantly different in prostate cancer of Caucasian, African-American and Japanese patients. Prostate 71: 489-497, 2011

15. Dong J, Xiao L, Sheng L, Xu J and Sun ZQ: TMPRSS2:ETS fusions and clinicopathologic characteristics of prostate cancer patients from Eastern China. Asian Pac J Cancer Prev 15: 3099-3103, 2014

16. Kessenbrock K, Plaks V and Werb Z: Matrix metalloproteinases: Regulators of the tumor microenvironment. Cell 141: 52-67, 2010.

17. Wang D, Li Q, Li K, Xiao P and Yin R: Twist-related protein 1-mediated regulation of mesenchymal change contributes to the migration and invasion of cervical cancer cells. Oncol Lett 10: 3107-3112, 2015.

18. Shi Z, Li X, Wu D, Tang R, Chen R, Xue S and Sun X: Silencing of HMGA2 suppresses cellular proliferation, migration, invasion, and epithelial-mesenchymal transition in bladder cancer. Tumour Biol 37: 7515-7523, 2016.

19. Li XW, Tuergan M and Abulizi G: Expression of MAPK1 in cervical cancer and effect of MAPK1 gene silencing on epithelial-mesenchymal transition, invasion and metastasis. Asian Pac J Trop Med 8: 937-943, 2015.

20. Banerjee $\mathrm{K}$ and Resat $\mathrm{H}$ : Constitutive activation of STAT3 in breast cancer cells: A review. Int J Cancer 138: 2570-2578, 2016.
21. Kato T, Fujita Y, Nakane K, Kojima T, Nozawa Y, Deguchi T and Ito M: ETS1 promotes chemoresistance and invasion of paclitaxel-resistant, hormone-refractory PC3 prostate cancer cells by up-regulating MDR1 and MMP9 expression. Biochem Biophys Res Commun 417: 966-971, 2012.

22. Qi M, Liu Z, Shen C, Wang L, Zeng J, Wang C, Li C, Fu W, Sun Y and Han B: Overexpression of ETV4 is associated with poor prognosis in prostate cancer: Involvement of uPA/uPAR and MMPs. Tumour Biol 36: 3565-3572, 2015.

23. Tomlins SA, Laxman B, Varambally S, Cao X, Yu J, Helgeson BE, Cao Q, Prensner JR, Rubin MA, Shah RB, et al: Role of the TMPRSS2-ERG gene fusion in prostate cancer. Neoplasia 10: 177-188, 2008.

24. Trusolino L and Comoglio PM: Scatter-factor and semaphorin receptors: Cell signalling for invasive growth. Nat Rev Cancer 2: 289-300, 2002.

25. Gabrovska PN, Smith RA, Tiang T, Weinstein SR, Haupt LM and Griffiths LR: Semaphorin-plexin signalling genes associated with human breast tumourigenesis. Gene 489: 63-69, 2011.

26. Ye S, Hao X, Zhou T, Wu M, Wei J, Wang Y, Zhou L, Jiang X, Ji L, Chen Y, et al: Plexin-B1 silencing inhibits ovarian cancer cell migration and invasion. BMC Cancer 10: 611, 2010.

27. Sun QP, Li LY, Chen Z, Pang J, Yang WJ, Zhou XF, Qiu JG, Su ZL, He D and Gao X: Detection of TMPRSS2-ETS fusions by a multiprobe fluorescence in situ hybridization assay for the early diagnosis of prostate cancer: A pilot study. J Mol Diagn 12: 718-724, 2010.

28. Perner S, Mosquera JM, Demichelis F, Hofer MD, Paris PL, Simko J, Collins C, Bismar TA, Chinnaiyan AM, De Marzo AM, et al: TMPRSS2-ERG fusion prostate cancer: An early molecular event associated with invasion. Am J Surg Pathol 31: 882-888, 2007.

29. Mehra R, Tomlins SA, Shen R, Nadeem O, Wang L, Wei JT, Pienta KJ, Ghosh D, Rubin MA, Chinnaiyan AM, et al: Comprehensive assessment of TMPRSS2 and ETS family gene aberrations in clinically localized prostate cancer. Mod Pathol 20: 538-544, 2007.

30. Font-Tello A, Juanpere N, de Muga S, Lorenzo M, Lorente JA, Fumado L, Serrano L, Serrano S, Lloreta J and Hernández S: Association of ERG and TMPRSS2-ERG with grade, stage, and prognosis of prostate cancer is dependent on their expression levels. Prostate 75: 1216-1226, 2015. 